\title{
Comparison of the power between microsatellite and single-nucleotide polymorphism markers for linkage and linkage disequilibrium mapping of an electrophysiological phenotype
} Hsiu-Fen Lin ${ }^{1,3}$, Suh-Hang Hank Juo ${ }^{1,2}$ and Rong Cheng*1

Address: ${ }^{1}$ Columbia Genome Center, Columbia University, New York, NY, USA, 2 Department of Epidemiology, Columbia University, New York, NY, USA and ${ }^{3}$ Department of Neurology, Kaohsiung Medical University, Kaohsiung, Taiwan

Email: Hsiu-Fen Lin - hl2199@columbia.edu; Suh-Hang Hank Juo - shj34@columbia.edu; Rong Cheng* - rc2116@columbia.edu

* Corresponding author

from Genetic Analysis Workshop 14: Microsatellite and single-nucleotide polymorphism

Noordwijkerhout, The Netherlands, 7-10 September 2004

Published: 30 December 2005

BMC Genetics 2005, 6(Suppl I):S7 doi:10.1 186/147I-2156-6-SI-S7

\begin{abstract}
We performed linkage and linkage disequilibrium (LD) mapping analyses to compare the power between microsatellite and single nucleotide polymorphism (SNP) markers. Chromosome-wide analyses were performed for a quantitative electrophysiological phenotype, ttth I, on chromosome 7. Multipoint analysis of microsatellite markers using the variance component (VC) method showed the highest LOD score of 4.20 at 162 cM, near D7S509 (163.7 cM). Two-point analysis of SNPs using the VC method yielded the highest LOD score of 3.98 in the lllumina SNP data and 3.45 in the Affymetrix SNP data around I52-153 cM. In family-based single SNP and SNP haplotype LD analysis, we identified seven SNPs associated with ttth I. We searched for any potential candidate genes in the location of the seven SNPs. The SNPs rs 1476640 and rs768055 are located in the FLJ40852 gene (a hypothetical protein), and SNP rs 1859646 is located in the TAS2R5 gene (a taste receptor). The other four SNPs are not located in any known or annotated genes. We found the high density SNP scan to be superior to microsatellites because it is effective in downstream fine mapping due to a better defined linkage region. Our study proves the utility of high density SNP in genome-wide mapping studies.
\end{abstract}

\section{Background}

Current strategy for complex disease gene mapping usually includes three stages. A genome-wide scan using microsatellite markers is performed to identify interesting chromosomal regions harboring the susceptibility loci. Then fine mapping is used as a follow-up to confirm and narrow the interesting regions. Finally, single nucleotide polymorphism (SNPs) are used to further saturate the regions and discover the candidate genes.

Genetic Analysis Workshop 14 (GAW14) provided data from the Collaborative Study on the Genetics of Alcoholism (COGA), including genome-wide microsatellite markers, genome-wide SNPs and several alcoholism- related phenotypes. This data allowed us to compare the power to detect susceptibility loci between SNPs and microsatellite markers in the context of genome-wide linkage and linkage disequilibrium (LD) analyses. We particularly chose a quantitative electrophysiological phenotype, $t$ tth 1 (the data from the Visual Oddball Experiment, measured from far frontal left side channel), as our phenotype of interest because a strong linkage signal was previously detected on chromosome 7 [1]. In this study, we restricted our focus to chromosome 7 rather than a genome-wide search. First, we performed chromosome-wide linkage analysis using microsatellite markers and high density SNPs. We then conducted family-based LD mapping analyses using each single SNP and SNP haplotypes. 

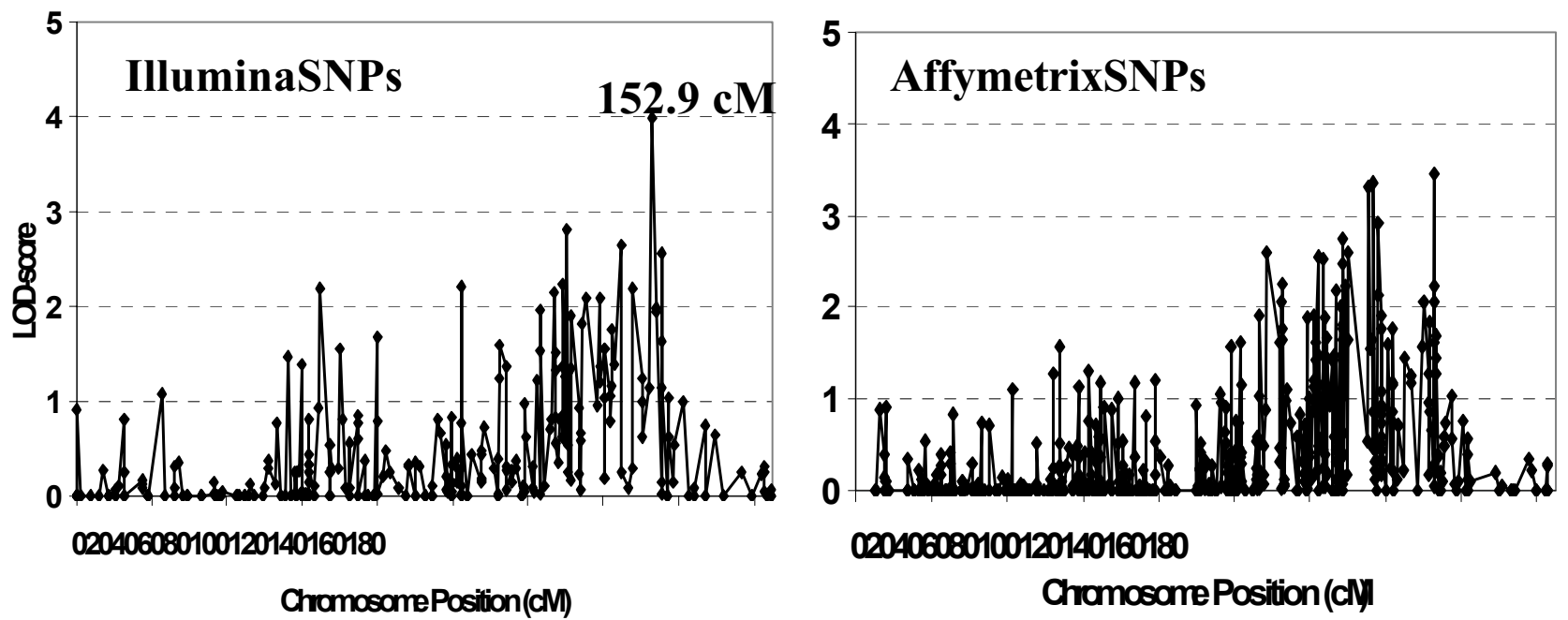

Figure I

Microsatellite multipoint VC analysis for ttth I (chromosome 7). Microsatellite marker multipoint VC analyses for quantitative trait ttthl on chromosome 7.

\section{Methods}

The COGA data set provided to GAW14 includes 1,350 members with genotype and phenotype information in 143 families. We used the quantitative data of ttth 1 , microsatellite markers, and two SNP panels (Illumina and Affymetrix panels) on chromosome 7 for our linkage and LD mapping analyses. First, a total of 31 microsatellite markers, at average inter-marker distance of $6.23 \mathrm{cM}$ on chromosome 7, were used for chromosome-wide scan to identify the interesting regions for ttth1. Two-point and multipoint analyses of microsatellite markers were con-

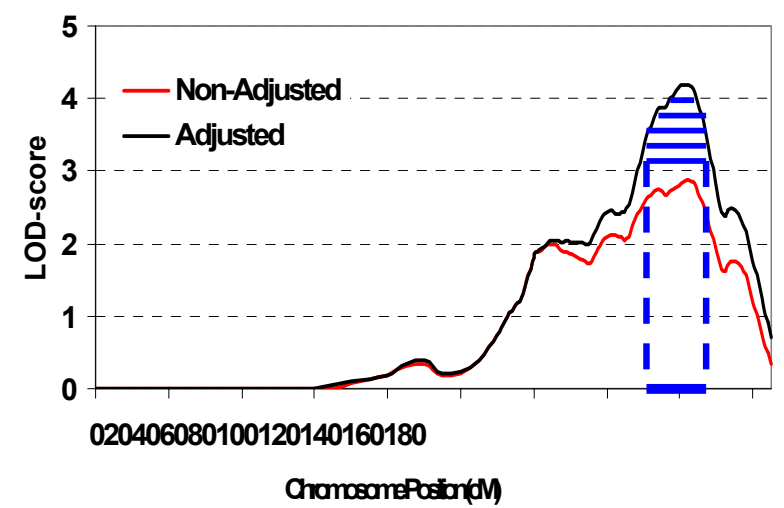

Figure 2

Age- and sex-adjusted SNP two-point VC analysis for ttth I on chromosome 7. Age- and sex-adjusted SNP twopoint VC analyses for quantitative trait ttth I on chromosome 7. ducted using the variance component (VC) method implemented in the SOLAR [2] and MERLIN programs [3]. Second, the 271 SNPs from the Illumina panel and 578 SNPs from the Affymetrix panel were used for twopoint VC analysis using MERLIN. Third, the FBAT program [4] was employed to perform the family-based LD analyses using single SNPs and SNP haplotypes. We used MERLIN to check for recombination between the tightly linked SNPs, and HAPLOVIEW [5] to estimate the linkage disequilibrium statistics ( $\left.\mathrm{D}^{\prime}\right)$ as well as the haplotype blocks. SNPs without recombination within haplotype blocks were used to create haplotypes for LD analysis.

\section{Results}

For microsatellite markers, two-point analysis showed the highest LOD score at D7S509 (163.7 cM): unadjusted $\mathrm{LOD}=2.70$ and age- and sex-adjusted LOD $=3.83$. The highest age- and sex-adjusted multipoint LOD score was 4.20 at $162 \mathrm{cM}$, with a 1-LOD support interval between 150 and $168 \mathrm{cM}$ (Figure 1). The marker closest to the multipoint highest LOD score was D7S509 at $163.7 \mathrm{cM}$.

For SNP markers, we found the highest two-point unadjusted LOD score of 3.87, and age- and sex-adjusted twopoint LOD score 3.98 at rs $940864(152.90 \mathrm{cM})$ in the Illumina SNP panel (Figure 2). In the Affymetrix panel, the highest two-point unadjusted LOD score was 2.93, and age- and sex-adjusted two-point LOD score was 3.45 at tsc0063156 (152.94 cM) (Figure 2). Multipoint age- and sex-adjusted analyses were also carried out for Illumina and Affymetrix panels, and the highest LOD scores, 3.70 $(152 \mathrm{cM})$ and $3.13(151 \mathrm{cM})$, were found for Illumina and 
Table I: Family-based single SNP LD analysis

\begin{tabular}{|c|c|c|c|c|c|c|}
\hline SNP name & Frequency & Map position (cM) & $\begin{array}{l}\text { Physical position } \\
\text { (bp) }\end{array}$ & z-score & $p$-value & Source \\
\hline rs|476640 & $0.448 / 0.552$ & 150.2 & $1408 \mid 9587$ & 2.256 & 0.024088 & Illumina \\
\hline rs768055 & $0.525 / 0.475$ & 150.2 & | 40820328 & 2.585 & 0.007296 & Illumina \\
\hline rs 1859646 & $0.413 / 0.587$ & 150.2 & | 4090288| & 2.585 & 0.009735 & Illumina \\
\hline tsc00584I6 & $0.743 / 0.257$ & 153.036 & I 44232436 & 2.073 & 0.038212 & Affymetrix \\
\hline tsc05906/5 & $0.878 / 0.122$ & 153.912 & $|45| 4 \mid 684$ & 2.893 & 0.003819 & Affymetrix \\
\hline
\end{tabular}

A

Table 2: Family-based SNP haplotype LD analysis

\begin{tabular}{|c|c|c|c|c|}
\hline Haplotype & Frequency & $z$-score & $p$-value ${ }^{a}$ & 2 SNPs \\
\hline hl: 2 I & 0.423 & 2.692 & 0.007094 & Illumina's rs \\
\hline h2: 12 & 0.416 & -1.869 & 0.061690 & 1476640 \\
\hline h3: 22 & 0.156 & -1.175 & 0.240070 & rs768055 \\
\hline h4: I I & 0.005 & -0.761 & 0.446698 & Distance $=741 \mathrm{bp}$ \\
\hline Global-test & $\mathrm{DF}=3$ & $\chi^{2}=7.564$ & 0.055929 & $D^{\prime}=0.98$ \\
\hline Haplotype & Frequency & $z$-score & $p$-value & 2 SNPs \\
\hline hl: 2 I & 0.438 & -2.325 & 0.020064 & Illumina's \\
\hline h2: 12 & 0.371 & 3.060 & 0.002212 & rs768055 \\
\hline h3: 22 & 0.135 & -0.609 & $0.54250 \mathrm{I}$ & rs1859646 \\
\hline h4: I I & 0.056 & -0.715 & 0.474386 & Distance $=82,553 \mathrm{bp}$ \\
\hline Global-test & $\mathrm{DF}=3$ & $\chi^{2}=9.494$ & 0.023396 & $D^{\prime}=0.88$ \\
\hline Haplotype & Frequency & $z$-score & $p$-value & 3 SNPs \\
\hline hl: I 2 I & 0.379 & -2.060 & 0.039409 & Illumina's rs I476640 \\
\hline h2: 2 I 2 & 0.367 & 3.064 & 0.002186 & rs768055 \\
\hline h3: 222 & 0.095 & -0.981 & 0.326434 & rs1859646 \\
\hline h4: 22 I & 0.060 & -0.633 & 0.526774 & $\begin{array}{l}\text { Distance }=741 \& 82,553 \\
\text { bp }\end{array}$ \\
\hline h5: 2 I I & 0.054 & -0.524 & 0.600176 & \\
\hline h6: 122 & 0.039 & 0.217 & 0.828502 & \\
\hline Others & 0.005 & 1 & & \\
\hline Global-test & $\mathrm{DF}=6$ & $\chi^{2}=10.352$ & 0.110580 & \\
\hline Haplotype & Frequency & $z$-score & $p$-value & 2 SNPs \\
\hline hl: I 2 & 0.687 & 1.402 & 0.160879 & Affymetrix's \\
\hline h2: 2 I & 0.235 & -0.318 & 0.750300 & tsc0058416 \\
\hline h3: 22 & 0.048 & 0.447 & 0.654974 & tsc00584I8 \\
\hline h4: I I & 0.030 & -3.456 & 0.000549 & Distance $=148 \mathrm{bp}$ \\
\hline Global-test & $D F=3$ & $\chi^{2}=12.272$ & 0.006509 & $D^{\prime}=0.98$ \\
\hline Haplotype & Frequency & $z$-score & $p$-value & 2 SNPs \\
\hline hl: I 2 & 0.851 & 3.097 & 0.001958 & Affymetrix's \\
\hline h2: 2 I & 0.115 & -1.537 & 0.124173 & tsc0590615 \\
\hline h3: I I & 0.033 & -3.616 & 0.000300 & tsc 0590614 \\
\hline h4: 22 & 0.001 & l & & Distance $=14 \mathrm{bp}$ \\
\hline Global-test & $D F=2$ & $\chi^{2}=|5.66|$ & 0.000397 & $D^{\prime}=1.00$ \\
\hline
\end{tabular}

aBold text indicates significant $p$-value. 
fymetrix panels, respectively. In either Illumina or Affymetrix panels, the highest LOD score was located within the 1-LOD support interval identified by microsatellite multipoint analysis. Both of the results from the two SNP data sets confirmed that the susceptibility locus is located within the interval of $150-168 \mathrm{cM}$ and the most likely location is approximately $153 \mathrm{cM}$. The average information contents in the 1-LOD support region are as high as 0.889 and 0.935 for Illumina and Affymetrix SNPs, respectively. Within a 5-cM interval between 150.5 and $155.5 \mathrm{cM}$, i.e., both sides of the peak SNP LOD scores at $153 \mathrm{cM}$, there are 23 Affymetrix SNPs and 12 Illumina SNPs. We found 6 Affymetrix SNPs and 5 Illumina SNPs had LOD scores between 1.0 and 1.9, and 4 Affymetrix SNPs and 4 Illumina SNPs had LOD scores $>1.9$ (data not shown).

Our family-based LD analysis focused on the 1-LOD support interval. A total of 40 Affymetrix SNPs and 24 Illumina SNPs were in this interval. Single SNP LD analyses showed significant associations on three Illumina SNPs and two Affymetrix SNPs (Table 1). Haplotype blocks were generated by using the HAPLOVIEW's default algorithm (confidence intervals). Several haplotype blocks were found in these SNPs with D' ranging from 0.74 to 1.00. The maximum number of SNPs in haplotype blocks was three. In Table 2, we listed only haplotype blocks with significant results. The analyses of haplotypes including 2 or 3 Illumina SNPs, and haplotypes including 2 Affymetrix SNPs showed significant associations. The significant $p$-values for the common (frequency $>0.1$ ) haplotypes ranged from 0.002 to 0.007 . The significant haplotype blocks were located at $150.2 \mathrm{cM}$ (rs1476640, rs768055 and rs1859646), $153.036 \mathrm{cM}$ (tsc0058416 and tsc0058418), and $153.912 \mathrm{cM}$ (tsc0590614 and tsc0590615). Comparing the results of the single SNP analysis with SNP haplotype analyses, suggested that the risk-bearing haplotype can be primarily ascribed to one SNP. However, the flanking SNP in the haplotype also contributed additional information leading to a more significant $p$-value. We further searched the SNP database using CHIP Bioinformatics Tools http://snpper.chip.org/ to find related information of the three closely linked haplotype blocks. The search showed: rs1476640 and rs768055 are located in the introns of the FLJ40852 gene, which is a hypothetical protein with unknown gene function, and rs 1859646 is located in the intron of the TAS2R5 gene, which is a taste receptor. The biological relevance of the three SNPs (rs1476640, rs768055, and rs1859646) were unknown. The other four SNPs (tsc0058416, tsc0058418, tsc0590614, and tsc0590615) are not located in any known or annotated genes.

\section{Discussion}

One of the major advantages of using high-density SNPs over microsatellite markers for genome scans its effectiveness in downstream fine mapping due to a better defined critical region. Our analysis of microsatellite markers showed strong linkage evidence of ttth1 at D7S509 on chromosome 7. However, we could not find significant results for the SNPs near D7S509 (163.7 cM) by either linkage- or family-based LD analysis. Our joint SNP linkage and LD mapping pinpointed a critical region between 150 and $154 \mathrm{cM}$, which is much smaller than the 1-LOD region by microsatellite markers. Using two different SNP panels, we found that the highest LOD scores and their locations are very close. Using family-based single SNP and SNP haplotype LD analyses, we further identified seven SNPs associated with phenotype ttth1. Our results indicated that the haplotype analysis may be more power than single SNP LD mapping in this dataset. Among them, three SNPs (rs1476640, rs768055, and rs1859646) are located within two potential genes, FLJ40852 and TAS2R5. It is also noteworthy that the associated SNPs and SNP haplotypes directly under the peak of linkage that is more precisely indicated by SNP markers. Combining linkage and LD analysis approaches, our results suggest that microsatellite markers may be less powerful than SNP markers to indicate the critical region. In our SNP LD analysis, three regions showed association, and there is apparently LD within each region. The strongest LD occurred in the block with two SNPs, tsc0590615 and tsc0590614. A comparison of the two-SNP haplotype LD analysis and the three-SNP haplotype LD analysis did not reveal stronger association in the block of rs 1476640 , rs768055, and rs1859646. Here, it appears that including more SNPs may not increase the overall evidence for association.

Although MERLIN has the advantage of faster speed than SOLAR in analyzing SNP data, it cannot effectively handle large pedigrees when analyzing microsatellite markers. In this study, we had to increase the default 24 bits to 40 bits while using MERLIN for SNP analysis. In this way, we analyzed all families with MERLIN, but the bit increase is not unrestricted and it may be a problem for even larger pedigree sizes. While we obtained identical results from SOLAR and MERLIN, MERLIN provided results in several hours, while SOLAR required several days.

Three recent studies comparing SNP and microsatellite analysis reported findings similar to ours: high-density SNPs defined a better critical region than microsatellite markers [6-8]. John et al. [6] used both the $10 \mathrm{~K}$ Affymetrix SNP panel and 10-cM microsatellite markers to perform a whole-genome screen of multiplex families with rheumatoid arthritis (RA). Their study showed a good concordance between the SNP and microsatellite genome 
scans. More importantly, the HLA locus, the major RA susceptibility locus on chromosome 6, was better defined by the SNPs than microsatellite markers. Middleton et al. [7] also compared the Affymetrix SNP panel with microsatellite markers in bipolar families. They concluded that a high degree of correspondence existed between the two approaches in general, but that the high-density SNP panel provided more power to detect linkage, especially in regions where the information content and coverage of the microsatellite markers were relatively low and potentially insufficient to detect linkage signal. Similarly, Schaid et al.'s study [8] found that SNP analysis identified more linkage peaks with narrower widths than did microsatellite markers. Moreover, Schaid et al. [8] and Huang et al. [9] also found that multipoint analysis using tightly linked SNPs inflates LOD scores. Therefore, future linkage studies should use SNP without strong LD when performing multipoint analysis.

\section{Conclusion}

This study found that SNP panels provide sufficient meiotic information for linkage analysis. The high-density SNP genome scan is more effective for fine mapping and LD mapping due to a better definition of the linkage region. Multipoint analysis of microsatellite markers showed strong linkage evidence within a 1-LOD support interval from 150 to $168 \mathrm{cM}$ on chromosome 7. Twopoint analyses of SNPs showed the highest LOD scores of 3.98 and 3.45 around $153 \mathrm{cM}$ for Illumina and Affymetrix SNP data, respectively. We identified seven SNPs associated with tth 1 in the candidate region harboring potential susceptibility loci using family-based single SNP and SNP haplotype LD analysis.

\section{Abbreviations \\ COGA: Collaborative Study of the Genetics of Alcoholism}

\section{GAW14: Genetic Analysis Workshop 14}

LD: Linkage disequilibrium

RA: Rheumatoid arthritis

SNP: Single-nucleotide polymorphism

VC: Variance component

\section{Authors' contributions}

H-FL performed linkage analyses and summarized the results. $\mathrm{RC}$ was responsible for data management, familybased LD analyses and manuscript preparation. S-HHJ designed and oversaw the project and prepared the manuscript. All authors read and approved the final manuscript.

\section{Acknowledgements}

This research was partly supported by grant, ROI NS047655 (S-HHJ and RC). $\mathrm{H}$-FL is supported by the Kaohsiung Medical University fellowship training grant.

\section{References}

I. Porjesz B, Begleiter H, Wang K, Almasy L, Chorlian DB, Stimus AT, Kuperman S, O'Connor SJ, Rohrbaugh J, Bauer LO, et al.: Linkage and linkage disequilibrium mapping of ERP and EEG phenotypes. Biol Psychol 2002, 61:229-248.

2. Almasy L, Blangero J: Multipoint quantitative-trait linkage analysis in general pedigrees. Am J Hum Genet 1998, 62: I 198-I2II.

3. Abecasis GR, Cherny SS, Cookson WO, Cardon LR: MERLIN rapid analysis of dense genetic maps using sparse gene flow trees. Nat Genet 2002, 30:97-I0I.

4. Horvath S, Xu X, Lake SL, Silverman EK, Weiss ST, Laird NM: Family-based tests for associating haplotypes with general phenotype data: application to asthma genetics. Genet Epidemiol 2004, 26:61-69.

5. Barrett JC, Fry B, Maller J, Daly MJ: Haploview: analysis and visualization of LD and haplotype maps. Bioinformatics 2005, 21:263-265.

6. John S, Shephard N, Liu G, Zeggini E, Cao M, Chen W, Vasavda N, Mills T, Barton A, Hinks A, et al:: Whole-genome scan, in a complex disease, using II,245 single-nucleotide polymorphisms: comparison with microsatellites. Am J Hum Genet 2004, 75:54-64.

7. Middleton FA, Pato MT, Gentile KL, Morley CP, Zhao X, Eisener AF, Brown A, Petryshen TL, Kirby AN, Medeiros H, et al:: Genomewide linkage analysis of bipolar disorder by use of a high-density single-nucleotide-polymorphism (SNP) genotyping assay: a comparison with microsatellite marker assays and finding of significant linkage to chromosome 6q22. Am J Hum Genet 2004, 74:886-897.

8. Schaid DJ, Guenther JC, Christensen GB, Hebbring S, Rosenow C, Hilker CA, McDonnell SK, Cunningham JM, Slager SL, Blute ML, Thibodeau SN: Comparison of microsatellites versus singlenucleotide polymorphisms in a genome linkage screen for prostate cancer-susceptibility Loci. Am J Hum Genet 2004, 75:948-965

9. Huang $\mathrm{Q}$, Shete $\mathrm{S}$, Amos $\mathrm{Cl}$ : Ignoring linkage disequilibrium among tightly linked markers induces false-positive evidence of linkage for affected sib pair analysis. Am J Hum Genet 2004, 75: II06-III2.

\begin{tabular}{|} 
Publish with Bio Med Central and every \\
scientist can read your work free of charge \\
"BioMed Central will be the most significant development for \\
disseminating the results of biomedical research in our lifetime. " \\
Sir Paul Nurse, Cancer Research UK \\
Your research papers will be: \\
• available free of charge to the entire biomedical community \\
• peer reviewed and published immediately upon acceptance \\
• cited in PubMed and archived on PubMed Central \\
• yours - you keep the copyright \\
Submit your manuscript here: \\
http://www.biomedcentral.com/info/publishing_adv.asp
\end{tabular}

